\title{
Robust Filtering, Prediction, Smoothing, and Observability of Uncertain Systems
}

\author{
S. O. Reza Moheimani, Member, IEEE, Andrey V. Savkin, and Ian R. Petersen, Senior Member, IEEE
}

\begin{abstract}
This paper is concerned with a class of continuoustime uncertain systems which satisfy a certain Integral Quadratic Constraint. The problems of robust filtering, robust prediction, and robust smoothing for such systems are defined, and nonconservative solutions are given in terms of Riccati differential equations. This paper also addresses a problem of robust observability for this class of uncertain systems.
\end{abstract}

Index Terms - Integral quadratic constraint, robust filtering, robust observability.

\section{INTRODUCTION}

A N IMPORTANT area of linear systems theory has been the theory of Kalman filtering, prediction, and smoothing. This theory has had a significant impact on control theory and signal processing, e.g., see [1]. In practice, to apply the standard Kalman filter, predictor, or smoother, it is imperative to have an accurate signal model. If this is not the case, it is known that the performance of a Kalman filter can be poor (e.g., see [2]). Indeed, usually there will be some uncertain parameters present in the process model. This fact has motivated the study of robust filtering for uncertain systems.

This problem can be regarded as an extension of the standard Kalman filters to the case of uncertain systems. In recent years, the increased interest in robust and $\mathcal{H}_{\infty}$ control theory has led to the publication of a large number of papers addressing the robust state estimation problem (e.g., see [3]-[11]). In these papers, a number of approaches are considered to tackle the problem of robust state estimation. In [4], [5], [7], and [8], the approach taken is to minimize the worst case $\mathcal{H}_{\infty}$ norm of the transfer function from the noise inputs to the estimation error output. This approach is closely related to widely studied problems of $\mathcal{H}_{\infty}$ control and filtering (e.g., see [12], [13]). The approach taken in [3], [6], [14], [15], and [12] is concerned with constructing a state estimator which bounds the mean square estimation error. This approach is more closely related to the standard Kalman

Manuscript received April 17, 1996; revised May 5, 1997. This paper was recommended by Associate Editor R. Ober.

S. O. R. Moheimani was with Department of Electrical Engineering, Australian Defence Force Academy, Canberra, ACT 2600, Australia. He is now with the Department of Electrical and Computer Engineering, University of Newcastle, Newcastle, Australia (e-mail: reza@ee.newcastle.edu.au).

A. V. Savkin is with the Department of Electrical and Electronic Engineering, University of Western Australia, Nedlands 6009, Australia (e-mail: savkin@ee.uwa.edu.au).

I. R. Petersen is with the Department of Electrical Engineering, Australian Defence Force Academy, Canberra, ACT 2600, Australia (e-mail: irp@ee.adfa.oz.au).

Publisher Item Identifier S 1057-7122(98)01416-0. filtering problem and involves the use of a fixed quadratic Lyapunov function to establish an upper bound on the state estimation error covariance.

The robust estimation problem is concerned with estimating the unmeasureable state variables of an uncertain plant. Depending on the available measurement, the robust estimation problem may be categorized as robust filtering, robust prediction, or robust smoothing. This paper is aimed at addressing these problems as well as the problem of robust observability of uncertain systems.

In [16] Bertsekas and Rhodes gave a deterministic interpretation of Kalman filtering. The problem considered in [16] is as follows. Given output measurements from a time-varying linear system with noise inputs subject to an $\mathcal{L}_{2}$ norm bound, find the set of all states consistent with these measurements. The solution to this problem was shown to be an ellipsoid in state space which is defined by the standard Kalman filter equations. Hence, the results of [16] give a deterministic interpretation of the standard Kalman filtering.

This paper is built on the results of [16] to obtain robust state estimators for a class of uncertain systems. In [9] the problem of robust Kalman filtering is solved based on the results of [16]. In this paper we rederive these results for a more general class of uncertain systems. Indeed, as in [17], we consider uncertain systems which have a deterministic control input. Moreover, we address the problems of robust prediction and smoothing in the same setting. These are extensions of robust Kalman filtering problem.

The underlying plant is assumed to be linear, time-varying, and uncertain with uncertainty satisfying a certain integral quadratic constraint. The integral quadratic constraint considered in this paper is assumed to hold over a finite time interval and allows for uncertainty in the initial conditions of the system. This integral quadratic constraint is a generalization of the IQC of [16] to allow for uncertainty outputs. Also, the state estimation problem considered here is a modification of the problem considered in [16] to allow for the uncertainty in the process model.

In the robust filtering problem, the output measurements for $t \leq T$ are available and the set of possible states at time $T$ consistent with these measurements and the integral quadratic constraint is to be determined. In the robust prediction problem the measurements are available up to a time $\tau<T$ and the set of possible states at $T$ is to be sought. The robust smoothing problem, however, is concerned with determining the set of possible states at a time $\tau<T$ consistent with the measurements up to a time $T$ and the integral quadratic constraint. 
There are considerable applications for the filtering results of this paper. In any application of the standard Kalman filtering where uncertainties enter the dynamics of the systems, our results could be used if the uncertainty could be modeled in an appropriate way. One particular area which is currently under investigation is the application of robust Kalman filtering in direct torque control (DTC) of Induction motors [18].

A fundamental concept in linear systems theory is that of observability [4]. Observability is concerned with the problem of determining the state of a dynamic system from observations of the output and control vectors over a finite time period. A system without uncertainty is said to be observable if it is possible to determine the initial state from the observation of the output and control vectors over a finite time interval. The concept of observability was introduced by Kalman [5] and plays an important role in the design of state observers. In particular, the concept is useful in solving the problem of reconstructing unmeasureable state variables from measured outputs.

The concept of observability is well studied and understood in the case of linear systems without uncertainty. However, in most real world problems, the model of the process includes some uncertain parameters. This paper is partly aimed at addressing the problem of robust observability for a class of uncertain continuous-time systems. Indeed, we extend the definition of observability to the case of uncertain systems. The system is allowed to be time-varying and the uncertainty is assumed to satisfy an integral quadratic constraint. An uncertain system is defined to be robustly observable if the set of possible initial states corresponding to a measured noisy output over a finite period of time is bounded. This problem is closely related to the robust smoothing problem.

In the following sections, we will show that the state estimator of [16] can be considered as a special case of our robust state estimator. Indeed, if the uncertainty is removed from our process model, then the linear time-varying system of [16] will be obtained.

The results of this paper generalize the results of [9] and [19] to the case of robust filtering, prediction, smoothing, and observability for uncertain systems with a control input.

The rest of the paper continues as follows. In Section II $<$ we define the class of uncertain systems considered in this paper and define the problems of robust filtering, prediction, and smoothing. The uncertainty is assumed to satisfy an integral quadratic constraint. This is a rich class of uncertain systems. We show that uncertain norm bounded systems can be considered as a special case of such uncertainty. Section III contains our solution to the problem of robust filtering. The solution is given in terms of a differential matrix Riccati equation and the set of possible states is shown to be an ellipsoid. The problem of robust filtering for time-invariant uncertain systems is also addressed in the same section. The problem of robust prediction is solved in Section IV. This problem is solved by changing it into an appropriate filtering problem and using the robust filtering results. Section V addresses the problem of robust smoothing. Robust smoothing problems can be divided into three different categories, namely, robust fixed interval smoothing, robust fixed lag smoothing, and the robust fixed point smoothing problems. However, Section V addresses only the problem of robust fixed interval smoothing. The importance of this particular problem is due to the fact that it motivates us to define the important problem of robust observability of uncertain systems.

Section VI addresses the problem of robust observability of uncertain systems. The problem is to find the admissible set of initial conditions consistent with output measurements over a finite interval of time and an integral quadratic constraint. This is closely related to the problem considered in Section V. Necessary and sufficient conditions for robust observability are given in terms of existence of a solution to a differential matrix Riccati equation and the set of possible initial conditions is shown to be an ellipsoid. Section VII contains an illustrative example.

\section{The Robust Filtering, Prediction, AND SMOOThing PROBlems}

In this paper we consider a time-varying uncertain system described by

$$
\begin{aligned}
\dot{x}(t) & =A(t) x(t)+B_{1}(t) w(t)+B_{2}(t) u(t) \\
z(t) & =K(t) x(t)+G(t) u(t) \\
y(t) & =C(t) x(t)+v(t)
\end{aligned}
$$

where $x(t) \in \mathbf{R}^{n}$ is the state, $w(t) \in \mathbf{R}^{p}$ and $v(t) \in \mathbf{R}^{l}$ are the uncertainty inputs, $u(t) \in \mathbf{R}^{h}$ is a known input, $z(t) \in \mathbf{R}^{q}$ is the uncertainty output, and $y(t) \in \mathbf{R}^{l}$ is the measured output; and $A(\cdot), B(\cdot), K(\cdot)$ and $C(\cdot)$ are bounded piecewise continuous matrix functions.

\section{A. System Uncertainty}

The uncertainty in the above system is described by an equation of the form

$$
\left[\begin{array}{c}
w(t) \\
v(t)
\end{array}\right]=\phi\left(t,\left.x(\cdot)\right|_{0} ^{t},\left.u(\cdot)\right|_{0} ^{t}\right)
$$

where the following integral quadratic constraint is satisfied. Let $X_{0}=X_{0}^{\prime}>0$ be a given matrix, $x_{0} \in \mathbf{R}^{n}$ be a given vector, $d>0$ be a given constant, and $Q(\cdot)=Q(\cdot)^{\prime}$ and $R(\cdot)=R(\cdot)^{\prime}$ be given bounded piecewise continuous matrix weighting functions satisfying the following condition. There exists a constant $\delta>0$ such that

$$
Q(t) \geq \delta I, \quad R(t) \geq \delta I
$$

for all $t$. Then for a given finite time interval $[0, T]$, we will consider the uncertainty inputs $w(\cdot)$ and $v(\cdot)$ and initial conditions $x(0)$ such that

$$
\begin{aligned}
& \left(x(0)-x_{0}\right)^{\prime} X_{0}\left(x(0)-x_{0}\right)+\int_{0}^{T}\left(w(t)^{\prime} Q(t) w(t)\right. \\
& \left.\quad+v(t)^{\prime} R(t) v(t)\right) d t \leq d+\int_{0}^{T}\|z(t)\|^{2} d t .
\end{aligned}
$$

Note that this description of uncertainty allows for uncertainty in the initial conditions. This uncertainty is determined by $X_{0}$. Indeed, larger values of $X_{0}$ correspond to lower values of 
uncertainty in initial conditions. Hence, if the initial condition is known, then we let $X_{0} \rightarrow \infty$ to give $X_{0}^{-1}=0$.

The uncertain system (2.1), (2.4) is a modification of the linear system considered in [16]. Indeed, if we let $K(\cdot) \equiv 0$, that is, we remove the uncertainty output from $(2.1)$, then the linear system of [16] will be obtained with $u(\cdot) \equiv 0$.

The uncertain system (2.1), (2.4) allows for uncertainty satisfying a standard norm bound constraint. In this case the uncertain system would be described by the state equations

$$
\begin{aligned}
\dot{x}(t)= & {\left[A(t)+B_{1}(t) \Delta_{1}(t) K(t)\right] x(t) } \\
& +\left[B_{2}(t)+B_{1}(t) \Delta_{1}(t) G(t)\right] u(t) \\
y(t)= & {\left[C(t)+\Delta_{2}(t) K(t)\right] x(t)+\Delta_{2}(t) G(t) u(t) }
\end{aligned}
$$

where $\Delta_{1}(t)$ and $\Delta_{2}(t)$ are uncertainty matrices satisfying

$$
\left\|\left[\Delta_{1}(t)^{\prime} Q(t)^{\frac{1}{2}} \quad \Delta_{2}(t)^{\prime} R(t)^{\frac{1}{2}}\right]\right\| \leq 1 \quad \text { for all } t .
$$

Also, the initial conditions would be required to satisfy the inequality

$$
\left(x(0)-x_{0}\right)^{\prime} X_{0}\left(x(0)-x_{0}\right) \leq d .
$$

To verify that such uncertainty is admissible for the uncertain system (2.1), (2.4), let

$$
\begin{aligned}
w(t) & =\Delta_{1}(t)[K(t) x(t)+G(t) u(t)] \\
v(t) & =\Delta_{2}(t)[K(t) x(t)+G(t) u(t)]
\end{aligned}
$$

where $\left\|\left[\Delta_{1}(t)^{\prime} Q(t)^{\frac{1}{2}} \Delta_{2}(t)^{\prime} R(t)^{\frac{1}{2}}\right]\right\| \leq 1$ for all $t$. Then (2.4) is satisfied.

In this paper we are concerned with the problems of "robust filtering," "robust prediction," "robust smoothing," and "robust observability" defined below.

In the robust filtering problem, $y(t)=y_{0}(t)$ is a fixed measured output of the uncertain system (2.1), defined on the time interval $[0, T]$ and $u(t)=u_{0}(t)$ is a fixed measured input to the uncertain system defined on the same time interval. The problem is to find the set $X_{T}\left[x_{0},\left.u_{0}(\cdot)\right|_{0} ^{T},\left.y_{0}(\cdot)\right|_{0} ^{T}, d\right]$ of all possible states $x(T)$ at time $T$ for the uncertain system (2.1), (2.2) with uncertainty inputs and initial conditions satisfying the constraint (2.4) (see also [9], [19]). In the robust prediction problem, the output measurement $y(t)=y_{0}(t)$ is only available over $[0, T-q]$ where $0<q<T$ and the problem is to find the set $X_{T}\left[x_{0},\left.u_{0}(\cdot)\right|_{0} ^{T},\left.y_{0}(\cdot)\right|_{0} ^{T-q}, d\right]$ of all possible states $x(T)$ at time $T$ for the uncertain system (2.1), (2.2) with uncertainty inputs and initial conditions satisfying (2.4). In the robust smoothing problem, the output measurement $y(t)=y_{0}(t)$ is available over $[0, T]$ and the set $X_{T-q}\left[x_{0},\left.u_{0}(\cdot)\right|_{0} ^{T},\left.y_{0}(\cdot)\right|_{0} ^{T}, d\right]$ of all possible states $x(T-q)$ at time $T-q$ for the uncertain system (2.1), (2.2) with uncertainty inputs and initial conditions satisfying (2.4) is to be determined. The problem of robust observability is closely related to the robust smoothing problem. This problem will be defined and solved in Section VI.

Note that if we remove the uncertainty output by assuming that $K(\cdot) \equiv 0$, then the above problems reduce to those of [16].

To understand the motivation behind the problem explained above, consider a standard Kalman filtering problem depicted

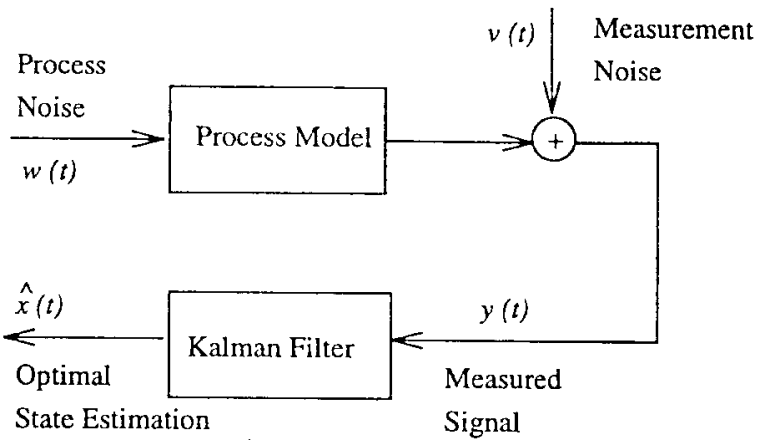

Fig. 1. A typical Kalman filtering problem.

in Fig. 1. The system is assumed to have the following dynamics:

$$
\begin{aligned}
& \dot{x}(t)=A x(t)+B w(t) \\
& y(t)=C x(t)+v(t)
\end{aligned}
$$

where $v(t)$ and $w(t)$ are white noise processes. A Kalman filter is then designed to give an optimal state estimation $\hat{x}(t)$. The procedure of designing a Kalman filter is well known (e.g., see [1]). This procedure requires a precise model of the system. A further assumption is that the spectral characteristics of the process and measurement noises are exactly known. In practice, however, these assumptions may not always be true.

In fact, we may not be certain about the spectral characteristics of the noise in our signal model. It is also possible that we may be considering a process model which is dependent on parameters whose values are imprecisely known due to manufacturing tolerances and variations with time or our process model may be a simplification of the true behavior of the process neglecting nonlinear effects and unmodeled dynamics. Therefore, we need a theory of Kalman filtering which allows for uncertainty in the process model and/or the spectral characteristic of the noise.

Consider the uncertain system of Fig. 2. Here, $v(t)$ and $w(t)$ are noise inputs (not necessarily random white Gaussian) and $\Delta$ represents the uncertain dynamics of the system. Whatever the form of the uncertainty $\Delta$, it is typically a quantity which is unknown but bounded in magnitude in some way. In other words, we do not know the value of the uncertainty but we know how big it can be. If the uncertainty block consists of time-varying uncertain parameters $\Delta(t)$, then its norm could be bounded, i.e., $\|\Delta(t)\| \leq \mu$. If $\Delta(s)$ is an uncertain transfer function, we could bound its magnitude at all frequencies, i.e., $\|\Delta(j \omega)\| \leq \mu$ for all $\omega>0$. This amounts to a bound on the $\mathcal{H}_{\infty}$ norm of the transfer function $\Delta(s)$.

Now suppose we have a transfer function uncertainty block, as in Fig. 3. Using Parseval's theorem, it follows that the frequency domain bound

$$
\|\Delta(j \omega)\| \leq \mu \text { for all } \omega>0
$$

is equivalent to the time-domain bound

$$
\int_{0}^{T} \Psi(t)^{\prime} \Psi(t) d t \leq \int_{0}^{T} z(t)^{\prime} z(t) d t
$$




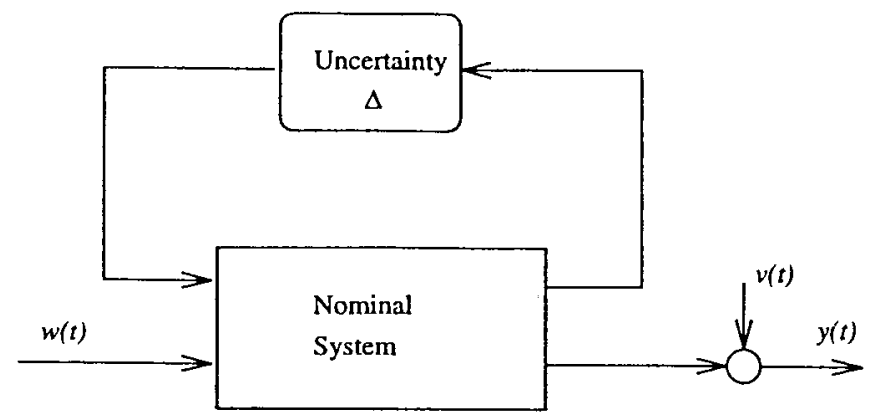

Fig. 2. Filtering problem when the system is uncertain.

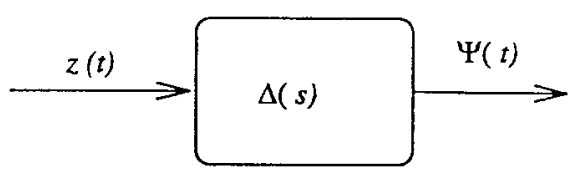

Fig. 3. The uncertain block.

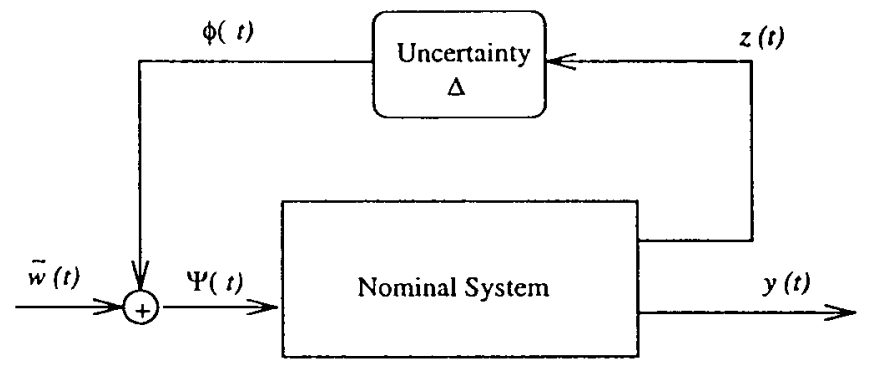

Fig. 4. Uncertain system with noise input.

for all signals $z(t)$. This time-domain uncertainty bound can be applied equally well to the case of a time-varying real uncertainty matrix $\Delta(t)$ or a nonlinear mapping.

The IQC uncertainty description can be extended to model the noise on the system as well as the uncertainty in the system dynamics, as shown in Fig. 4. To model both noise and uncertainty, we should modify the IQC to

$$
\int_{0}^{T} \Psi(t)^{\prime} \Psi(t) d t \leq d+\int_{0}^{T} z(t)^{\prime} z(t) d t
$$

where $d>0$ determines the bound on the size of the noise. To understand why the constant $d$ had to be added to the IQC, note that if $z(t)=0$, the uncertainty block $\Delta$ makes no contribution to signal $\Psi(t)$. However, $\Psi(t)$ can still be nonzero due to the presence of the noise signal. Introducing a positive $d$ avoids this difficulty. At this stage, it should be pointed out that this IQC modeling of noise corresponds to an energy bound on the noise rather than a stochastic white noise description.

If we have both process noise and measurement noise (as in Fig. 5), we can split the signal $\Psi$ into two components

$$
\Psi=\left[\begin{array}{c}
w(t) \\
v(t)
\end{array}\right] .
$$

Then the IQC becomes

$$
\int_{0}^{T}\left[w(t)^{\prime} Q(t) w(t)+v(t)^{\prime} R(t) v(t)\right] d t \leq d+\int_{0}^{T}\|z(t)\|^{2} d t
$$

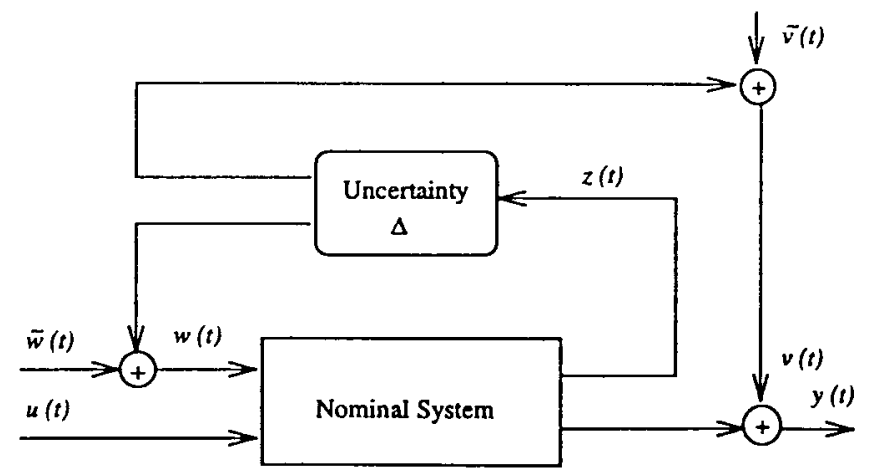

Fig. 5. Uncertain system with measurement noise and process noise.

where $Q(t)>0$ and $R(t)>0$ are weighting matrices. To encorporate uncertainty due to the initial conditions into the IQC, the term $\left(x(0)-x_{0}\right)^{\prime} X_{0}\left(x(0)-x_{0}\right)$ should be added to the left-hand side of the inequality. Therefore the general form of the IQC will be as given in (2.4).

\section{The Robust Filtering Problem}

Our solution to the robust filtering problem stated above requires the solution of the following Riccati differential equation:

$$
\begin{gathered}
-\dot{X}(t)=X(t) A(t)+A(t)^{\prime} X(t)+X(t) B_{1}(t) Q(t)^{-1} \\
\cdot B_{1}(t)^{\prime} X(t)+K(t)^{\prime} K(t)-C(t)^{\prime} R(t) C(t) \\
X(0)=X_{0} ; \quad t \in[0, T] .
\end{gathered}
$$

Also, we need to solve the following state equation:

$$
\begin{aligned}
\dot{\eta}(t)= & -\left[A(t)+B_{1}(t) Q(t)^{-1} B_{1}(t)^{\prime} X(t)\right]^{\prime} \eta(t) \\
& +C(t)^{\prime} R(t) y_{0}(t)+\left[K(t)^{\prime} G(t)\right. \\
& \left.+X(t) B_{2}(t)\right] u_{0}(t) ; \quad \eta(0)=X_{0} x_{0}
\end{aligned}
$$

for $t \in[0, T]$.

The following theorem is the main result of this section. It describes the set of all possible states of the uncertain system (2.1), (2.4) at time $T$ given the input $u(\cdot)$ and measurements of the output $y(\cdot)$ over the time interval $[0, T]$.

Theorem 3.1 Let $X_{0}=X_{0}^{\prime}>0$ be a given matrix and $Q(\cdot)=Q(\cdot)^{\prime}$ and $R(\cdot)=R(\cdot)^{\prime}$ be given matrix functions such that (2.3) holds on the time interval $[0, T]$. Then for a given vector $x_{0} \in \mathbf{R}^{n}$, a constant $d>0$, a fixed input $u(t)=u_{0}(t)$, and a fixed measured output $y(t)=y_{0}(t)$, the set $X_{T}\left[x_{0},\left.u_{0}(\cdot)\right|_{0} ^{T},\left.y_{0}(\cdot)\right|_{0} ^{T}, d\right]$ is bounded if and only if the Riccati equation (3.1) has a solution over $[0, T]$ such that $X(T)>0$. Furthermore, the set $X_{T}\left[x_{0},\left.u_{0}(\cdot)\right|_{0} ^{T},\left.y_{0}(\cdot)\right|_{0} ^{T}, d\right]$ is given by

$$
\begin{aligned}
& X_{T}\left[x_{0},\left.u_{0}(\cdot)\right|_{0} ^{T},\left.y_{0}(\cdot)\right|_{0} ^{T}, d\right] \\
& \quad=\left\{x_{T} \in \mathbf{R}^{n}: x_{T}^{\prime} X(T) x_{T}-2 x_{T}^{\prime} \eta(T)+h_{T} \leq d\right\}
\end{aligned}
$$

where

$$
\begin{aligned}
h_{T}= & x_{0}^{\prime} X_{0} x_{0}+\int_{0}^{T}\left\{y_{0}(t)^{\prime} R(t) y_{0}(t)\right. \\
& -u_{0}(t)^{\prime} G(t)^{\prime} G(t) u_{0}(t)-\eta(t)^{\prime} B_{1}(t) Q(t)^{-1} B_{1}(t)^{\prime} \eta(t) \\
& \left.+2 u_{0}(t)^{\prime} B_{2}(t) \eta(t)\right\} d t .
\end{aligned}
$$


Proof: Suppose that the output measurement $\left.y_{0}(\cdot)\right|_{0} ^{T}$ is given and $\left.u_{0}(\cdot)\right|_{0} ^{T}$ is known. From the definition of $X_{T}\left[x_{0},\left.u_{0}(\cdot)\right|_{0} ^{T},\left.y_{0}(\cdot)\right|_{0} ^{T}, d\right]$, it follows that

$$
x_{T} \in X_{T}\left[x_{0},\left.u_{0}(\cdot)\right|_{0} ^{T},\left.y_{0}(\cdot)\right|_{0} ^{T}, d\right]
$$

if and only if there exist vector functions $x(\cdot), w(\cdot)$, and $v(\cdot)$ defined on $[0, T]$ such that $x(T)=x_{T}$, the following inequality is satisfied:

$$
\begin{gathered}
\left(x(0)-x_{0}\right)^{\prime} X_{0}\left(x(0)-x_{0}\right)+\int_{0}^{T}\left[w(t)^{\prime} Q(t) w(t)\right. \\
\left.+v(t)^{\prime} R(t) v(t)\right] d t \leq d+\int_{0}^{T}\|z(t)\|^{2} d t
\end{gathered}
$$

and

$$
v(t)=y_{0}(t)-C(t) x(t), \quad \text { for all } t \in[0, T] .
$$

Substituting (3.6) into (3.5) implies that $x_{T} \in X_{T}\left[x_{0},\left.u_{0}(\cdot)\right|_{0} ^{T}\right.$, $\left.\left.y_{0}(\cdot)\right|_{0} ^{T}, d\right]$ if and only if there exist a $w(\cdot) \in \mathcal{L}_{2}[0, T]$ such that

$$
J\left[x_{T}, w(\cdot)\right] \leq d
$$

where $J\left[x_{T}, w(\cdot)\right]$ is defined by

$$
\begin{aligned}
& J\left[x_{T}, w(\cdot)\right]=\left(x(0)-x_{0}\right)^{\prime} X_{0}\left(x(0)-x_{0}\right) \\
& \quad+\int_{0}^{T}\left\{w(t)^{\prime} Q(t) w(t)-\left\|K(t) x(t)+G(t) u_{0}(t)\right\|^{2}\right. \\
& \left.\quad+\left(y_{0}(t)-C(t) x(t)\right)^{\prime} R(t)\left(y_{0}(t)-C(t) x(t)\right)\right\} d t .
\end{aligned}
$$

Now, let us assume that the set $X_{T}\left[x_{0},\left.u_{0}(\cdot)\right|_{0} ^{T},\left.y_{0}(\cdot)\right|_{0} ^{T}, d\right]$ is bounded. Consider the functional (3.8) with $x_{0}=0, u_{0}(\cdot) \equiv 0$, and $y_{0}(\cdot) \equiv 0$. In this case, $J$ is a homogeneous quadratic functional with a terminal cost term. Also, consider the set $X_{T}[0,0,0,1]$ corresponding to $x_{0}=0, u_{0}(\cdot) \equiv 0, y_{0}(\cdot) \equiv 0$, and $d=1$. Since $X_{T}[0,0,0,1]$ is bounded, there exists a constant $\alpha>0$ such that all vectors $x_{T} \in \mathbf{R}^{n}$ with $\left\|x_{T}\right\|=\alpha$ do not belong to the set $X_{T}[0,0,0,1]$. Hence,

$$
J\left[x_{T}, w(\cdot)\right]>1
$$

for all $x_{T} \in \mathbf{R}^{n}$ such that $\left\|x_{T}\right\|=\alpha$ and for all $w(\cdot) \in$ $\mathcal{L}_{2}[0, T]$. Since, $J$ is a homogeneous quadratic functional, we have $J\left[a x_{T}, a w(\cdot)\right]=a^{2} J\left[x_{T}, w(\cdot)\right]$ and (3.9) implies that

$$
\inf _{w(\cdot) \in \mathcal{L}_{2}[0, T]} J\left[x_{T}, w(\cdot)\right]>0
$$

for all $x_{T} \neq 0$.

The optimization problem (3.10) subject to the constraint defined by the system (2.1) is a linear quadratic optimal control problem in which time is reversed. In this linear quadratic optimal control problem, a sign indefinite quadratic cost function is being considered. Using a known result from linear quadratic optimal control theory, we conclude that condition (3.10) implies that there exists a solution $X(\cdot)$ to the Riccati equation (3.1) with initial condition $X(0)=X_{0}$ for all $t \in[0, T]$ such that $X(T)>0$; e.g., see page 23 of [20]. This proves the first part of the theorem.

To prove the second part of the theorem note that we have already shown that $x_{T} \in X_{T}\left[x_{0},\left.u_{0}(\cdot)\right|_{0} ^{T},\left.y_{0}(\cdot)\right|_{0} ^{T}, d\right]$ if and only if there exists a $w(\cdot) \in \mathcal{L}_{2}[0, T]$ such that inequality (3.7) is satisfied. Now, consider the following optimization problem

$$
\inf _{w(\cdot) \in \mathcal{L}_{2}[0, T]} J\left[x_{T}, w(\cdot)\right]
$$

where the minimum is taken over all $x(\cdot)$ and $w(\cdot)$ connected by (2.1) with the boundary condition $x(T)=x_{T}$. The minimization problem (3.11) can be considered as a linear quadratic optimal tracking problem in which the system operates in reverse time. To convert this tracking problem into a tracking problem of the form considered in [21] and [16], we decompose the state into $x(t)=\tilde{x}(t)+x_{1}(t)$ where $x_{1}(t)$ is the solution to the state equation

$$
\dot{x}_{1}(t)=A(t) x_{1}(t)+B_{2}(t) u_{0}(t) ; \quad x_{1}(0)=0 .
$$

It follows from (2.1) and (3.12) that $\tilde{x}(t)$ satisfies the state equation

$$
\dot{\tilde{x}}(t)=A(t) \tilde{x}(t)+B_{1}(t) w(t)
$$

with $\tilde{x}(0)=x(0)$. Therefore, the cost function (3.8) can be rewritten as

$$
\begin{aligned}
& J\left[x_{T}, w(\cdot)\right]=\tilde{J}\left[\tilde{x}_{T}, w(\cdot)\right]=\left(x(0)-x_{0}\right)^{\prime} X_{0}\left(x(0)-x_{0}\right) \\
& \quad+\int_{0}^{T}\left\{w(t)^{\prime} Q(t) w(t)-\| K(t)\left[\tilde{x}(t)+x_{1}(t)\right]\right. \\
& \quad+G(t) u_{0}(t) \|^{2}+\left(y_{0}(t)-C(t)\left[\tilde{x}(t)+x_{1}(t)\right]\right)^{\prime} \\
& \left.\quad \times R(t)\left(y_{0}(t)-C(t)\left[\tilde{x}(t)+x_{1}(t)\right]\right)\right\} d t
\end{aligned}
$$

where $\tilde{x}(T)=\tilde{x}_{T}=x_{T}-x_{1}(T)$. It can be observed that (3.13) and (3.14) define a linear quadratic tracking problem similar to the one considered in [21] with $y_{0}(\cdot), u_{0}(\cdot)$, and $x_{1}(\cdot)$, all treated as reference inputs. In fact, the only difference between the standard linear quadratic optimal tracking problem and the tracking problem considered here is that in our case the cost is sign indefinite. The solution to this tracking problem is well known; e.g., see [21, Section 3.6]. Indeed, if the matrix function $X(\cdot)$ is the solution to Riccati equation (3.1) then the infimum in (3.11) will be achieved for any $x_{0}, u_{0}(\cdot)$, and any $y_{0}(\cdot)$. Furthermore, as in [21], we can write

$$
\min _{w(\cdot) \in \mathcal{L}_{2}[0, T]} J\left[\tilde{x}_{T}, w(\cdot)\right]=\tilde{x}_{T}^{\prime} X(T) \tilde{x}_{T}-2 \tilde{x}_{T}^{\prime} v(T)+g(T)
$$

where $v(t)$ and $g(t)$ are solutions of the following differential equations:

$$
\begin{aligned}
\dot{v}(t)= & -\left[A(t)+B_{1}(t) Q(t)^{-1} B_{1}(t)^{\prime} X(t)\right]^{\prime} v(t) \\
& +C(t)^{\prime} R(t) y_{0}(t)+K(t)^{\prime} G(t) u_{0}(t) \\
& +\left[K(t)^{\prime} K(t)-C(t)^{\prime} R(t) C(t)\right] x_{1}(t) \\
& v(0)=X_{0} x_{0} \\
\dot{g}(t)= & {\left[y_{0}(t)-C(t) x_{1}(t)\right]^{\prime} R(t)\left[y_{0}(t)-C(t) x_{1}(t)\right] } \\
& -\left\|G(t) u_{0}(t)+K(t) x_{1}(t)\right\|^{2} \\
& -v(t)^{\prime} B_{1}(t) Q(t)^{-1} B_{1}(t)^{\prime} v(t) \quad g(0)=x_{0}^{\prime} X_{0} x_{0}
\end{aligned}
$$

for $t \in[0, T]$. Here, $x_{1}(t)$ is the solution of the following state equation:

$$
\dot{x}_{1}(t)=A(t) x_{1}(t)+B_{2}(t) u_{0}(t), \quad x_{1}(0)=0
$$

for $t \in[0, T]$. 
Now, let us define

$$
\eta(t)=v(t)+X(t) x_{1}(t)
$$

and

$$
h(t)=g(t)+2 x_{1}(t)^{\prime} \eta(t)-x_{1}(t)^{\prime} X(t) x_{1}(t) .
$$

It can be verified that $\eta(t)$ satisfies (3.2) and $h(t)$ satisfies the following differential equation:

$$
\begin{aligned}
\dot{h}(t)= & y_{0}(t)^{\prime} R(t) y_{0}(t)-\eta(t)^{\prime} B_{1}(t) Q(t)^{-1} B_{1}(t)^{\prime} \eta(t) \\
& -u_{0}(t)^{\prime} G(t)^{\prime} G(t) u_{0}(t)+2 u_{0}(t)^{\prime} B_{2}(t) \eta(t)
\end{aligned}
$$

for $t \in[0, T]$ and with $h(0)=x_{0}^{\prime} X_{0} x_{0}$. Hence,

$$
\min _{w(\cdot) \in \mathcal{L}_{2}[0, T]} J\left[x_{T}, w(\cdot)\right]=x_{T}^{\prime} X(T) x_{T}-2 x_{T}^{\prime} v(T)+h_{T}
$$

where $h_{T}=h(T)$ is as in (3.4).

From this we can conclude that the set $X_{T}\left[x_{0},\left.u_{0}(\cdot)\right|_{0} ^{T}\right.$, $\left.\left.y_{0}(\cdot)\right|_{0} ^{T}, d\right]$ satisfies

$$
\begin{aligned}
& X_{T}\left[x_{0},\left.u_{0}(\cdot)\right|_{0} ^{T},\left.y_{0}(\cdot)\right|_{0} ^{T}, d\right] \\
& \quad=\left\{x_{T} \in \mathbf{R}^{n}: \min _{w(\cdot) \in \mathcal{L}_{2}[0, T]} J\left[x_{T}, w(\cdot)\right] \leq d\right\}
\end{aligned}
$$

which is the same as (3.3). This completes the proof of the theorem.

Remarks If the Riccati differential equation (3.1) has a positive definite solution over $[0, T]$ then the set $X_{T}\left[x_{0},\left.u_{0}(\cdot)\right|_{0} ^{T},\left.y_{0}(\cdot)\right|_{0} ^{T}, d\right]$ can be characterized as follows:

$$
\begin{aligned}
& X_{T}\left[x_{0},\left.u_{0}(\cdot)\right|_{0} ^{T},\left.y_{0}(\cdot)\right|_{0} ^{T}, d\right] \\
& =\left\{x_{T} \in \mathbf{R}^{n}:\left(x_{T}-\hat{x}(T)\right)^{\prime} P(T)^{-1}\left(x_{T}-\hat{x}(T)\right)\right. \\
& \left.\quad \leq d+\rho_{T}\right\}
\end{aligned}
$$

where $\hat{x}(t)$ is the solution to the following state equation with initial condition $\hat{x}(0)=x_{0}$ :

$$
\begin{aligned}
\dot{\hat{x}}(t)= & {\left[A(t)+P(t)\left[K(t)^{\prime} K(t)-C(t)^{\prime} R(t) C(t)\right]\right] \hat{x}(t) } \\
& +P(t) C(t)^{\prime} R(t) y_{0}(t) \\
& +\left[P(t) K(t)^{\prime} G(t)+B_{2}(t)\right] u_{0}(t)
\end{aligned}
$$

for $t \in[0, T]$. Here, $P(\cdot)$ is the positive definite solution of the Riccati differential equation

$$
\begin{aligned}
\dot{P}(t)= & A(t) P(t)+P(t) A(t)^{\prime} \\
& +P(t)\left[K(t)^{\prime} K(t)-C(t)^{\prime} R(t) C(t)\right] P(t) \\
& +B(t) Q(t)^{-1} B(t)^{\prime}
\end{aligned}
$$

for $t \in[0, T]$, with initial condition $P(0)=X_{0}^{-1}$. Moreover, $\rho_{T}$ is defined by

$$
\begin{aligned}
\rho_{T}=\int_{0}^{T} & \left\{\left\|K(t) \hat{x}(t)+G(t) u_{0}(t)\right\|^{2}-(C(t) \hat{x}(t)\right. \\
& \left.\left.-y_{0}(t)\right)^{\prime} R(t)\left(C(t) \hat{x}(t)-y_{0}(t)\right)\right\} d t .
\end{aligned}
$$

This can be verified by defining $\hat{x}(t)$ as $\hat{x}(t)=P(t) \eta(t)$ with $P(\cdot)=X(\cdot)^{-1}$ being the solution to (3.17) over $[0, T]$ and completing the square in (3.3) and using (3.17), (3.2), and (3.16).
In the above equations, if we set $K(\cdot) \equiv 0$ and $u_{0}(\cdot) \equiv 0$, then our results will reduce to those of [16]. This is because with these assumptions, the uncertain system (2.1) and (2.4) reduces to the linear system considered in [16].

Note that the Riccati differential equation (3.17) is similar to the Riccati differential equation arising in $\mathcal{H}_{\infty}$ filtering problems; e.g., see [22, Ch. 7]. However, the state estimator proposed here is different from the $\mathcal{H}_{\infty}$ state estimator due to the presence of the term $P(t) K(t)^{\prime} K(t)$.

We now consider the robust filtering problem for an uncertain time-invariant system. In this problem, we consider the following time-invariant uncertain system defined over the time interval $[0, T]$ :

$$
\begin{aligned}
\dot{x}(t) & =A x(t)+B_{1} w(t)+B_{2} u(t) \\
z(t) & =K x(t)+G u(t) \\
y(t) & =C x(t)+v(t)
\end{aligned}
$$

where $x(t) \in \mathbf{R}^{n}$ is the state, $w(t) \in \mathbf{R}^{p}$ and $v(t) \in \mathbf{R}^{l}$ are the uncertainty inputs, $z(t) \in \mathbf{R}^{q}$ is the uncertainty output, $y(t) \in \mathbf{R}^{l}$ is the measured output, and $u(t) \in \mathbf{R}^{h}$ is a known input. The uncertainty in this system is defined as follows. Let $Q=Q^{\prime}>0$ and $R=R^{\prime}>0$ be given matrices associated with the system (3.19). Then, given a finite time interval $[0, T]$, the uncertainty inputs and initial conditions for this uncertain system are required to satisfy the following integral quadratic constraint:

$$
\begin{aligned}
& \left(x(0)-x_{0}\right)^{\prime} X_{0}\left(x(0)-x_{0}\right)+\int_{0}^{T}\left(w(t)^{\prime} Q w(t)\right. \\
& \left.\quad+v(t)^{\prime} R v(t)\right) d t \leq d+\int_{0}^{T}\|z(t)\|^{2} d t .
\end{aligned}
$$

Theorem 3.2 Consider the uncertain system (3.19), (3.20) defined on $[0, T]$ with weighting matrices $Q=Q^{\prime}>0$ and $R=R^{\prime}>0$ and suppose that the pair $(A, B)$ is stabilizable. If the algebraic Riccati equation

$$
A P+P A^{\prime}+P\left[K^{\prime} K-C^{\prime} R C\right] P+B Q^{-1} B^{\prime}=0
$$

has a solution $\hat{P}>0$ such that the matrix $\left[A^{\prime}-\left[C^{\prime} R C-\right.\right.$ $\left.\left.K^{\prime} K\right] \hat{P}\right]$ is stable and $\hat{P} \geq X_{0}^{-1}$, then for any matrix $X_{0}=X_{0}^{\prime}>0$ such that $X_{0}^{-1} \leq \hat{P}$, the set $X_{T}\left[x_{0},\left.u_{0}(\cdot)\right|_{0} ^{T}\right.$, $\left.\left.y_{0}(\cdot)\right|_{0} ^{T}, d\right]$ is bounded and described by

$$
\begin{aligned}
X_{T}\left[x_{0},\left.u_{0}(\cdot)\right|_{0} ^{T},\left.y_{0}(\cdot)\right|_{0} ^{T}, d\right] & \\
= & \left\{x_{T} \in \mathbf{R}^{n}:\left(x_{T}-\hat{x}(T)\right)^{\prime} P(T)^{-1}\left(x_{T}-\hat{x}(T)\right)\right. \\
& \left.\leq d+\rho_{T}\right\} .
\end{aligned}
$$

Here, $P(\cdot)$ is the solution of the following Riccati differential equation:

$$
\begin{aligned}
\dot{P}(t)= & A P(t)+P(t) A^{\prime}+P(t)\left[K^{\prime} K\right. \\
& \left.-C^{\prime} R C\right] P(t)+B Q^{-1} B^{\prime}
\end{aligned}
$$

for $t \in[0, T]$ with initial condition $P(0)=X_{0}^{-1}$. Also $\hat{x}(t)$ is the solution to the following state equation with initial condition $\hat{x}(0)=x_{0}$ :

$$
\begin{aligned}
\dot{\hat{x}}(t)= & {\left[A+P(t)\left[K^{\prime} K-C^{\prime} R C\right]\right] \hat{x}(t) } \\
& +P(t) C^{\prime} R y_{0}(t)+\left[P(t) K^{\prime} G+B_{2}\right] u_{0}(t)
\end{aligned}
$$


for $t \in[0, T]$. Furthermore, the constant $\rho_{T}$ is defined as follows:

$$
\begin{aligned}
\rho_{T}=\int_{0}^{T} & {\left[\left\|K \hat{x}(t)+G u_{0}(t)\right\|^{2}-(C \hat{x}(t)\right.} \\
& \left.\left.-y_{0}(t)\right)^{\prime} R\left(C \hat{x}(t)-y_{0}(t)\right)\right] d t .
\end{aligned}
$$

Moreover, $P(\cdot)$ is defined on $[0, \infty)$ and has the property $P(t) \rightarrow \hat{P}$ as $t \rightarrow \infty$.

Proof: This follows directly from Theorem 3.1 and Theorem 2.2 of [9].

At this stage it should be pointed out that the robust filter designed using the above procedure is nonconservative for an uncertain system with an Integral Quadratic Contraint. As explained earlier, the IQC description of uncertainty includes other more restrictive uncertainty descriptions. If the above procedure is to be used to design a robust filter for such an uncertain system in which the uncertainty is more accurately modeled by a more restrictive uncertainty description (for example a system with norm bounded uncertainty), it will result in a conservative design.

\section{The Robust Prediction Problem}

In this section, we are concerned with the problem of "robust prediction" as defined in Section II. In this problem, unlike the robust filtering case, the output measurement $y(\cdot)$ is only available for $0 \leq t \leq T-q$ and the set of possible states at time $T$ corresponding to this measurement and the uncertain system (2.1), (2.4) is to be determined.

To prove the main result of this section, we change the robust prediction problem into a special robust filtering problem. This enables us to derive necessary and sufficient conditions similar to those of the previous section.

Our solution to the robust prediction problem requires the solution to the following Riccati differential equation:

$$
\begin{aligned}
-\dot{X}(t)= & X(t) A(t)+A(t)^{\prime} X(t) \\
& +X(t) B_{1}(t) Q(t)^{-1} B_{1}(t)^{\prime} X(t)+K(t)^{\prime} K(t) \\
& -C(t)^{\prime} S(t) C(t) ; \quad X(0)=X_{0} ; \quad t \in[0, T]
\end{aligned}
$$

Also, we need to solve the following state equation:

$$
\begin{aligned}
\dot{\eta}(t)= & -\left[A(t)+B_{1}(t) Q(t)^{-1} B_{1}(t)^{\prime} X(t)\right]^{\prime} \eta(t) \\
& +C(t)^{\prime} S(t) y_{0}(t)+\left[K(t)^{\prime} G(t)+X(t) B_{2}(t)\right] u_{0}(t)
\end{aligned}
$$

for $t \in[0, T-q]$ with $\eta(0)=X_{0} x_{0}$. Here,

$$
S(t) \triangleq \begin{cases}R(t), & 0 \leq t \leq T-q \\ 0, & T-q<t \leq T\end{cases}
$$

The following theorem is the main result of this section. It describes the set of all possible states of the uncertain system (2.1), (2.4) at time $T$ given measurements of the output $y(\cdot)$ over the time interval $[0, T-q]$.
Theorem 4.1 Let $X_{0}=X_{0}^{\prime}>0$ be a given matrix, and $Q(\cdot)=Q(\cdot)^{\prime}$ and $R(\cdot)=R(\cdot)^{\prime}$ be given matrix functions such that condition (2.3) holds on the time interval $[0, T]$. Then, for a given vector $x_{0} \in \mathbf{R}^{n}$, a constant $d>0$, a fixed input $u(t)=u_{0}(t)$, and a fixed measured output $y(t)=y_{0}(t)$, the set $X_{T}\left[x_{0},\left.u_{0}(\cdot)\right|_{0} ^{T},\left.y_{0}(\cdot)\right|_{0} ^{T-q}, d\right]$ is bounded if and only if the Riccati equation (4.1) has a solution over $[0, T]$ such that $X(T)>0$. Furthermore, the set $X_{T}\left[x_{0},\left.u_{0}(\cdot)\right|_{0} ^{T},\left.y_{0}(\cdot)\right|_{0} ^{T-q}, d\right]$ is given by

$$
\begin{aligned}
& X_{T}\left[x_{0},\left.u_{0}(\cdot)\right|_{0} ^{T},\left.y_{0}(\cdot)\right|_{0} ^{T-q}, d\right] \\
& \quad=\left\{x_{T} \in \mathbf{R}^{n}: x_{T}^{\prime} X(T) x_{T}-2 x_{T}^{\prime} \eta(T)+h_{T} \leq d\right\}
\end{aligned}
$$

where

$$
\begin{aligned}
h_{T}= & x_{0}^{\prime} X_{0} x_{0}+\int_{0}^{T-q} y_{0}(t)^{\prime} R(t) y_{0}(t) d t \\
& +\int_{0}^{T}\left[2 u_{0}(t)^{\prime} B_{2}(t) \eta(t)-\eta(t)^{\prime} B_{1}(t) Q(t)^{-1} B_{1}(t)^{\prime} \eta(t)\right. \\
& \left.\quad-u_{0}(t)^{\prime} G(t)^{\prime} G(t) u_{0}(t)\right] d t
\end{aligned}
$$

Proof: Here we only sketch a proof of the theorem. Note that if output measurement $\left.y_{0}(\cdot)\right|_{0} ^{T-q}$ is given and $\left.u_{0}(\cdot)\right|_{0} ^{T}$ is known, then from the definition of $X_{T}\left[x_{0},\left.u_{0}(\cdot)\right|_{0} ^{T}\right.$, $\left.\left.y_{0}(\cdot)\right|_{0} ^{T-q}, d\right]$, it follows that

$$
x_{T} \in X_{T}\left[x_{0},\left.u_{0}(\cdot)\right|_{0} ^{T},\left.y_{0}(\cdot)\right|_{0} ^{T-q}, d\right]
$$

if and only if there exist vector functions $x(\cdot)$, and $w(\cdot)$ defined on $[0, T]$, and $v(\cdot)$ defined on $[0, T-q]$ such that $x(T)=x_{T}$, the following inequality is satisfied:

$$
\begin{array}{r}
\left(x(0)-x_{0}\right)^{\prime} X_{0}\left(x(0)-x_{0}\right)+\int_{0}^{T} w(t)^{\prime} Q(t) w(t) d t \\
+\int_{0}^{T-q} v(t)^{\prime} R(t) v(t) d t \leq d+\int_{0}^{T}\|z(t)\|^{2} d t
\end{array}
$$

and

$$
v(t)=y_{0}(t)-C(t) x(t) \text { for all } t \in[0, T-q]
$$

Substituting (4.7) into (4.6) and using the identification (4.3) implies that $x_{T} \in X_{T}\left[x_{0},\left.u_{0}(\cdot)\right|_{0} ^{T},\left.y_{0}(\cdot)\right|_{0} ^{T-q}, d\right]$ if and only if there exist a $w(\cdot) \in \mathcal{L}_{2}[0, T]$ such that

$$
J\left[x_{T}, w(\cdot)\right] \leq d
$$

where $J\left[x_{T}, w(\cdot)\right]$ is defined by

$$
\begin{aligned}
J\left[x_{T}, w(\cdot)\right]= & \left(x(0)-x_{0}\right)^{\prime} X_{0}\left(x(0)-x_{0}\right) \\
& +\int_{0}^{T}\left\{w(t)^{\prime} Q(t) w(t)\right. \\
& -\left\|K(t) x(t)+G(t) u_{0}(t)\right\|^{2}\left(y_{0}(t)\right. \\
& \left.-C(t) x(t))^{\prime} S(t)\left(y_{0}(t)-C(t) x(t)\right)\right\} d t .
\end{aligned}
$$

The rest of the theorem can be proved along the same lines as Theorem 3.1. 


\section{The Robust Fixed Interval Smoothing Problem}

In this section, we present and discuss our solution to the robust smoothing problem as defined in Section II. The robust smoothing problem may be divided into three categories: the robust fixed interval smoothing, the robust fixed lag smoothing, and the robust fixed point smoothing problems. In this section, we only concentrate on the robust fixed point smoothing problem and comment that the other two problems may be defined and solved in the same manner.

The reason behind our interest in the robust fixed interval smoothing is that it gives us an intuition to consider an important problem, namely the robust observability of the uncertain system. This problem will be discussed in the next section and its relation to the smoothing problem considered in this section will be clarified.

Our solution involves the following Riccati differential equations:

$$
\begin{aligned}
-\dot{X}(t)= & X(t) A(t)+A(t)^{\prime} X(t) \\
& +X(t) B_{1}(t) Q(t)^{-1} B_{1}(t)^{\prime} X(t)+K(t)^{\prime} K(t) \\
& -C(t)^{\prime} R(t) C(t) ; \quad X(0)=X_{0} \\
-\dot{Y}(t)= & Y(t) A(t)+A(t)^{\prime} Y(t) \\
& -Y(t) B_{1}(t) Q(t)^{-1} B_{1}(t)^{\prime} Y(t)-K(t)^{\prime} K(t) \\
& +C(t)^{\prime} R(t) C(t) ; \quad Y(T)=0 .
\end{aligned}
$$

It will also include a solution to the differential equations:

$$
\begin{aligned}
\dot{\eta}(t)= & -\left[A(t)+B_{1}(t) Q(t)^{-1} B_{1}(t)^{\prime} X(t)\right]^{\prime} \eta(t) \\
& +C(t)^{\prime} R(t) y_{0}(t)+\left[K(t)^{\prime} G(t)\right. \\
& \left.+X(t) B_{2}(t)\right] u_{0}(t) ; \quad \eta(0)=X_{0} x_{0}
\end{aligned}
$$

for $t \in[0, T-q]$ and

$$
\begin{aligned}
-\dot{\xi}(t)= & {\left[A(t)-B(t) Q(t)^{-1} B(t)^{\prime} Y(t)\right]^{\prime} \xi(t) } \\
& +C(t)^{\prime} R(t) y_{0}(t)-\left[Y(t) B_{2}(t)\right. \\
& \left.-K(t)^{\prime} G(t)\right] u_{0}(t) ; \quad \xi(T)=0
\end{aligned}
$$

for $t \in[T-q, T]$.

Note that (5.1) and (5.3) must be solved forward in time while (5.2) and (5.4) must be solved in reverse time. This is due to the nature of the robust smoothing problem which will be clarified later.

The following theorem is the main result of this section. It describes the set of all possible states for the uncertain system (2.1), (2.4) at time $T-q$ given measurements of the output $y(\cdot)$ over the time interval $[0, T]$.

Theorem 5.1 Let $X_{0}=X_{0}^{\prime}>0$ be a given matrix, and $Q(\cdot)=Q(\cdot)^{\prime}$ and $R(\cdot)=R(\cdot)^{\prime}$ be given matrix functions such that condition (2.3) holds on the time interval $[0, T]$. Also assume that the Riccati differential equation (5.1) has a solution over time interval $t \in[0, T-q]$ such that $X(T-q)>$ 0 and the Riccati differential equation (5.2) has a solution over time interval $t \in[T-q, T]$ such that $Y(T-q)>0$. Then, for a given vector $x_{0} \in \mathbf{R}^{n}$, a constant $d>0$ and a fixed measured output $y(t)=y_{0}(t)$, the set $X_{T-q}\left[x_{0},\left.u_{0}(\cdot)\right|_{0} ^{T},\left.y_{0}(\cdot)\right|_{0} ^{T}, d\right]$ is bounded. Furthermore, the set $X_{T-q}\left[x_{0},\left.u_{0}(\cdot)\right|_{0} ^{T},\left.y_{0}(\cdot)\right|_{0} ^{T}, d\right]$ is given by

$$
\begin{aligned}
X_{T-q} & {\left[x_{0},\left.y_{0}(\cdot)\right|_{0} ^{T}, d\right] } \\
= & \left\{x_{T-q} \in \mathbf{R}^{n}: x_{T-q}^{\prime} X(T-q) x_{T-q}\right. \\
& -2 x_{T-q}^{\prime} \eta(T-q)+h_{T-q}+x_{T-q}^{\prime} Y(T-q) x_{T-q} \\
& \left.-2 x_{T-q}^{\prime} \xi(T-q)+s_{T-q} \leq d\right\}
\end{aligned}
$$

where $\eta(t)$ and $\xi(t)$ are solutions to (5.3) and (5.4) and

$$
\begin{aligned}
h_{T-q}= & x_{0}^{\prime} X_{0} x_{0}+\int_{0}^{T-q}\left\{y_{0}(t)^{\prime} R(t) y_{0}(t)-u_{0}(t)^{\prime} G(t)^{\prime}\right. \\
& \times G(t) u_{0}(t)-\eta(t)^{\prime} B_{1}(t) Q(t)^{-1} B_{1}(t)^{\prime} \eta(t) \\
& \left.+2 u_{0}(t)^{\prime} B_{2}(t) \eta(t)\right\} d t
\end{aligned}
$$

and

$$
\begin{aligned}
s_{T-q}= & \int_{0}^{T-q}\left\{y_{0}(t)^{\prime} R(t) y_{0}(t)-u_{0}(t)^{\prime} G(t)^{\prime} G(t) u_{0}(t)\right. \\
& -\xi(t)^{\prime} B_{1}(t) Q(t)^{-1} B_{1}(t)^{\prime} \xi(t) \\
& \left.-2 u_{0}(t)^{\prime} B_{1}(t) \xi(t)\right\} d t
\end{aligned}
$$

Proof: Suppose that the output measurement $\left.y_{0}(\cdot)\right|_{0} ^{T}$ is given and the input $\left.u_{0}(\cdot)\right|_{0} ^{T}$ is known. From the definition of $X_{T-q}\left[x_{0}, u_{0}(\cdot),\left.y_{0}(\cdot)\right|_{0} ^{T}, d\right]$, it follows that

$$
x_{T-q} \in X_{T-q}\left[x_{0},\left.u_{0}(\cdot)\right|_{0} ^{T},\left.y_{0}(\cdot)\right|_{0} ^{T}, d\right]
$$

if and only if there exist vector functions $x(\cdot), w(\cdot)$, and $v(\cdot)$ defined on $[0, T]$ such that there exist a solution to (2.1) satisfying $x(T-q)=x_{T-q}$, the following inequality is satisfied:

$$
\begin{aligned}
& \left(x(0)-x_{0}\right)^{\prime} X_{0}\left(x(0)-x_{0}\right)+\int_{0}^{T}\left[w(t)^{\prime} Q(t) w(t) d t\right. \\
& \left.\quad+v(t)^{\prime} R(t) v(t)\right] d t \leq d+\int_{0}^{T}\|z(t)\|^{2} d t
\end{aligned}
$$

and

$$
v(t)=y_{0}(t)-C(t) x(t) \text { for all } t \in[0, T] .
$$

Substituting (5.9) into (5.8) implies that

$$
x_{T-q} \in X_{T-q}\left[x_{0},\left.u_{0}(\cdot)\right|_{0} ^{T},\left.y_{0}(\cdot)\right|_{0} ^{T}, d\right]
$$

if and only if there exist a $w(\cdot) \in \mathcal{L}_{2}[0, T]$ such that

$$
J_{1}\left[x_{T-q}, w(\cdot)\right]+J_{2}\left[x_{T-q}, w(\cdot)\right] \leq d
$$

where $J_{1}\left[x_{T-q}, w(\cdot)\right]$ is defined by

$$
\begin{aligned}
& J_{1}\left[x_{T-q}, w(\cdot)\right] \\
&=\left(x(0)-x_{0}\right)^{\prime} X_{0}\left(x(0)-x_{0}\right)+\int_{0}^{T-q}\left\{w(t)^{\prime} Q(t) w(t)\right. \\
&-\left\|K(t) x(t)+G(t) u_{0}(t)\right\|^{2}+\left(y_{0}(t)-C(t) x(t)\right)^{\prime} R(t) \\
&\left.\times\left(y_{0}(t)-C(t) x(t)\right)\right\} d t .
\end{aligned}
$$

and $J_{2}\left[x_{T-q}, w(\cdot)\right]$ is defined by

$$
\begin{aligned}
& J_{2}\left[x_{T-q}, w(\cdot)\right] \\
& =\int_{T-q}^{T}\left\{w(t)^{\prime} Q(t) w(t)-\left\|K(t) x(t)+G(t) u_{0}(t)\right\|^{2}\right. \\
& \left.\quad+\left(y_{0}(t)-C(t) x(t)\right)^{\prime} R(t)\left(y_{0}(t)-C(t) x(t)\right)\right\} d t
\end{aligned}
$$


and $x(\cdot)$ is the solution to $(2.1)$ with input $w(\cdot)$ and boundary condition $x(T-q)=x_{T-q}$.

Now consider the following minimization problem:

$$
\min _{w(\cdot) \in \mathcal{L}_{2}[0, T]}\left\{J_{1}\left[x_{T-q}, w(\cdot)\right]+J_{2}\left[x_{T-q}, w(\cdot)\right]\right\}
$$

where the minimum is taken over all $x(\cdot)$ and $w(\cdot)$ connected by (2.1) with the boundary condition $x(T-q)=x_{T-q}$. Since for each $x_{T-q}, J_{1}\left[x_{T-q}, w(\cdot)\right]$ depends only on the portion of $w(\cdot)$ defined over the time interval $[0, T-q]$ and $J_{2}\left[x_{T-q}, w(\cdot)\right]$ depends on the portion of $w(\cdot)$ defined over the time interval $[T-q, T],(5.13)$ may be written as

$$
\min _{w(\cdot) \in \mathcal{L}_{2}[0, T-q]} J_{1}\left[x_{T-q}, w(\cdot)\right]+\min _{w(\cdot) \in \mathcal{L}_{2}[T-q, T]} J_{2}\left[x_{T-q}, w(\cdot)\right] .
$$

The minimization of $J_{1}\left[x_{T-q}, w(\cdot)\right]$ is a linear quadratic tracking problem in reverse time over time interval $[0, T-q]$. The minimization of $J_{2}\left[x_{T-q}, w(\cdot)\right]$ is another linear quadratic optimization problem, but in forward time over the time interval $[T-q, T]$. To convert these tracking problems into tracking problems considered in [16] and [21], we use the same technique as in the proof of Theorem 3.1. That is, we decompose the state into $x(t)=\tilde{x}(t)+x_{1}(t)$ where $x_{1}(\cdot)$ is the solution of the state equation (3.12) over $[0, T]$. Hence, we may rewrite (3.12) and (5.12) as

$$
\begin{aligned}
J_{1}\left[x_{T-q}, w(\cdot)\right]= & \tilde{J}_{1}\left[\tilde{x}_{T-q}, w(\cdot)\right] \\
= & \left(x(0)-x_{0}\right)^{\prime} X_{0}\left(x(0)-x_{0}\right) \\
& +\int_{0}^{T-q}\left\{w(t)^{\prime} Q(t) w(t)-\| K(t)[\tilde{x}(t)\right. \\
& \left.+x_{1}(t)\right]+G(t) u_{0}(t) \|^{2}+\left(y_{0}(t)\right. \\
& \left.-C(t)\left[\widetilde{x}(t)+x_{1}(t)\right]\right)^{\prime} R(t)\left(y_{0}(t)\right. \\
& \left.\left.-C(t)\left[\tilde{x}(t)+x_{1}(t)\right]\right)\right\} d t
\end{aligned}
$$

and

$$
\begin{aligned}
J_{2}\left[x_{T-q}, w(\cdot)\right]= & \tilde{J}_{2}\left[\tilde{x}_{T-q}, w(\cdot)\right] \\
= & \int_{T-q}^{T}\left\{w(t)^{\prime} Q(t) w(t)-\| K(t)[\tilde{x}(t)\right. \\
& \left.+x_{1}(t)\right]+G(t) u_{0}(t) \|^{2}+\left(y_{0}(t)\right. \\
& \left.-C(t)\left[\tilde{x}(t)+x_{1}(t)\right]\right)^{\prime} R(t)\left(y_{0}(t)\right. \\
& \left.\left.-C(t)\left[\tilde{x}(t)+x_{1}(t)\right]\right)\right\} d t .
\end{aligned}
$$

With this transformation, the minimization of (5.13) has been converted into

$$
\min _{w(\cdot) \in \mathcal{L}_{2}[0, T]}\left\{\tilde{J}_{1}\left[x_{T-q}, w(\cdot)\right]+\tilde{J}_{2}\left[x_{T-q}, w(\cdot)\right]\right\} \cdot
$$

The minimization of $\tilde{J}_{1}\left[x_{T-q}, w(\cdot)\right]$ is a linear quadratic tracking problem in reverse time over time interval $[0, T-$ $q]$ while minimization of $\tilde{J}_{2}\left[x_{T-q}, w(\cdot)\right]$ is another linear quadratic optimization problem in forward time over the time interval $[T-q, T]$. In these tracking problems $u_{0}(\cdot), y_{0}(\cdot)$ and $x_{1}(\cdot)$ are all treated as reference inputs. Solvability of these tracking problems is guaranteed by the assumptions made in the theorem. That is, the Riccati differential equations (5.1) and (5.2) have solutions such that $X(T-q)>0$ and $Y(T-q)>0$. Indeed, using a well-known result on the linear quadratic tracking problem, (e.g., see [21]), it follows that a minimum in (5.14) will be achieved for any $x_{0}, u_{0}(\cdot)$ and any $y_{0}(\cdot)$. Furthermore, as in [16] and [9], we can write

$$
\begin{aligned}
\min _{w(\cdot) \in \mathcal{L}_{2}[0, T-q]} J_{1}\left[x_{T-q}, w(\cdot)\right]+\min _{w(\cdot) \in \mathcal{L}_{2}[T-q, T]} J_{2}\left[x_{T-q}, w(\cdot)\right] \\
=x_{T-q}^{\prime} X(T-q) x_{T-q}-2 x_{T-q}^{\prime} \eta(T-q)+h_{T-q} \\
\quad+x_{T-q}^{\prime} Y(T-q) x_{T-q}-2 x_{T-q}^{\prime} \xi(T-q)+s_{T-q}
\end{aligned}
$$

where $\eta(T-q)$ and $\xi(T-q)$ are defined by the solutions to differential equations (5.3) and (5.4) and $h_{T-q}$ and $s_{T-q}$ are defined by (5.6) and (5.7). Also, $X(T-q)$ and $Y(T-q)$ are defined by (5.1) and (5.2). From this, we can conclude that the set

$$
\begin{aligned}
X_{T-q}\left[x_{0},\left.y_{0}(\cdot)\right|_{0} ^{T}, d\right] \\
=\left\{x_{T} \in \mathbf{R}^{n}: \min _{w(\cdot) \in \mathcal{L}_{2}[0, T-q]} J_{1}\left[x_{T-q}, w(\cdot)\right]\right. \\
\left.\quad+\min _{w(\cdot) \in \mathcal{L}_{2}[T-q, T]} J_{2}\left[x_{T-q}, w(\cdot)\right] \leq d\right\}
\end{aligned}
$$

is as given in (5.5). This completes the proof of the theorem.

Note that the solution to the robust smoothing problem is generated by two filters, one operating in forward time and the other operating in reverse time. The filter operating forward in time corresponds to the tracking problem in reverse time while the other filter corresponds to the tracking problem in forward time. A similar situation occurs in the stochastic optimal smoothing of linear systems, e.g., see [23, Section 3.8].

\section{The Robust Observability Problem}

This section is concerned with a problem of robust observability for uncertain systems of the form (2.1) which satisfy the following integral quadratic constraint:

$$
\begin{aligned}
& \int_{0}^{T}\left\{w(t)^{\prime} Q(t) w(t)+v(t)^{\prime} R(t) v(t)\right\} d t \\
& \quad \leq d+\int_{0}^{T}\|z(t)\|^{2} d t
\end{aligned}
$$

for a given $T$. Note that the integral quadratic constraint (6.1) is different from (2.4). Indeed, in (6.1) the matrix $X_{0}$ is assumed to be zero. Recall that $X_{0}$ is a measure of our knowledge of the initial conditions $x(0)$. The smaller the $X_{0}$, the more uncertain the initial conditions are. In other words, the assumption $X_{0}=0$ is equivalent to assuming that no a priori information exists about the initial condition $x(0)$. Hence, the set of possible initial conditions given a measured output could be unbounded. This motivates our definition of robust observability.

Definition 6.1 The uncertain system (2.1), (6.1) is said to be robustly observable on the time interval $[0, T]$ if for any given $u_{0}(\cdot)$ and $y_{0}(\cdot)$ and any constant $d>0$ the corresponding set $X_{0}\left[\left.u_{0}(\cdot)\right|_{0} ^{T},\left.y_{0}(\cdot)\right|_{0} ^{T}, d\right]$ of all possible states at $t=0$ is bounded.

This definition of robust observability extends the standard definition of observability for time-varying linear systems to the case of systems with uncertainty. The robust observability problem can be considered as a special case of the robust smoothing problem. Indeed, in Section $\mathrm{V}$, if we let $X_{0} \rightarrow 0$, 
and $q \rightarrow T$, then the robust smoothing problem will be equivalent to the robust observability problem considered here. Hence, we should be able to use Theorem 5.1 directly. However, in the sequel, we will show that a stronger result can be obtained by considering robust observability as an independent problem.

Theorem 6.1 is the main result of this section. It describes our solution to the robust observability problem. This solution involves the Riccati differential equation

$$
\begin{aligned}
-\dot{Y}(t)= & Y(t) A(t)+A(t)^{\prime} Y(t) \\
& -Y(t) B_{1}(t) Q(t)^{-1} B_{1}(t)^{\prime} Y(t)-K(t)^{\prime} K(t) \\
& +C(t)^{\prime} R(t) C(t) ; \quad Y(T)=0
\end{aligned}
$$

and the following differential equation:

$$
\begin{aligned}
-\dot{\phi}(t)= & {\left[A(t)-B_{1}(t) Q(t)^{-1} B_{1}(t)^{\prime} Y(t)\right]^{\prime} \phi(t) } \\
& +C(t)^{\prime} R(t) y_{0}(t)-\left[Y(t) B_{2}(t)\right. \\
& \left.-K(t)^{\prime} G(t)\right] u_{0}(t) ; \quad \phi(T)=0
\end{aligned}
$$

for $t \in[0, T]$.

Theorem 6.1 Let $Q(\cdot)=Q(\cdot)^{\prime}$ and $R(\cdot)=R(\cdot)^{\prime}$ be given matrix functions such that $(2.2)$ holds on the time interval $[0, T]$. Consider the uncertain system $(2.1),(6.1)$. Then the following statements are equivalent.

(i) For a given constant $d>0$, the uncertain system (2.1), (6.1) is robustly observable.

(ii) The Riccati equation (5.2) with terminal condition $Y(T)=0$ has a solution over time interval $t \in[0, T]$ such that $Y(0)>0$.

Moreover, if condition (ii) holds, then

$$
X_{0}\left[\left.u_{0}(\cdot)\right|_{0} ^{T},\left.y_{0}(\cdot)\right|_{0} ^{T}, d\right]=x_{0}^{\prime} Y(0) x_{0}-2 x_{0}^{\prime} \phi(0)+s_{0} \leq d
$$

where $\phi(\cdot)$ is defined by (6.3) and

$$
\begin{aligned}
s_{0}= & \int_{0}^{T}\left\{y_{0}(t)^{\prime} R(t) y_{0}(t)-u_{0}(t)^{\prime} G(t)^{\prime} G(t) u_{0}(t)\right. \\
& -\phi(t)^{\prime} B_{1}(t) Q(t)^{-1} B_{1}(t)^{\prime} \phi(t) \\
& \left.-2 u_{0}(t)^{\prime} B_{1}(t)^{\prime} \phi(t)\right\} d t .
\end{aligned}
$$

Proof: (i) $\Rightarrow$ (ii) Given a measured output $y_{0}(\cdot)$ and an input $u_{0}(\cdot)$, we have by the definition that $x_{0} \in$ $X_{0}\left[\left.u_{0}(\cdot)\right|_{0} ^{T},\left.y_{0}(\cdot)\right|_{0} ^{T}, d\right]$ if and only if there exist vector functions $x(\cdot), w(\cdot)$ and $v(\cdot)$ satisfying (2.1) such that $x(0)=x_{0}$, the constraint (6.1) holds, and

$$
v(t)=y_{0}(t)-C(t) x(t) \text { for all } t \in[0, T] .
$$

Substitution of (6.6) into (6.1) implies that $x_{0} \in$ $X_{0}\left[\left.u_{0}(\cdot)\right|_{0} ^{T},\left.y_{0}(\cdot)\right|_{0} ^{T}, d\right]$ if and only if there exists an input $w(\cdot) \in \mathcal{L}_{2}[0, T]$ such that $J\left[x_{0}, w(\cdot)\right] \leq d$ where

$$
\begin{aligned}
& J\left[x_{0}, w(\cdot)\right] \\
& =\int_{0}^{T}\left\{w(t)^{\prime} Q(t) w(t)-\left\|K(t) x(t)+G(t) u_{0}(t)\right\|^{2}\right. \\
& \left.\quad+\left(y_{0}(t)-C(t) x(t)\right)^{\prime} R(t)\left(y_{0}(t)-C(t) x(t)\right)\right\} d t
\end{aligned}
$$

and $x(\cdot)$ is the solution to $(2.1)$ with input $w(\cdot)$ and boundary condition $x(0)=x_{0}$.

Now consider the functional (6.7) with $y_{0}(\cdot) \equiv 0$ and $u_{0}(\cdot) \equiv 0$. In this case, $J$ is a homogeneous quadratic functional. Let us consider the set $X_{0}[0,0,1]$ corresponding to $y_{0}(\cdot) \equiv 0, u_{0}(\cdot) \equiv 0$ and $d=1$. Since $X_{0}[0,0,1]$ is assumed to be bounded, a constant $\alpha>0$ can be found such that all vectors $x_{0} \in \mathbf{R}^{n}$ with $\left\|x_{0}\right\|=\alpha$ do not belong to the set $X_{0}[0,0,1]$. Therefore, $J\left[x_{0}, w(\cdot)\right]>1$ for all $x_{0} \in \mathbf{R}^{n}$ such that $\left\|x_{0}\right\|=\alpha$ and for all $w(\cdot) \in \mathcal{L}_{2}[0, T]$. This along with the fact that $J$ is a homogeneous quadratic functional implies that $J\left[a x_{0}, a w(\cdot)\right]=a^{2} J\left[x_{0}, w(\cdot)\right]$ and hence

$$
\inf _{w(\cdot) \subset \mathcal{L}_{2}[0, T]} J\left[x_{0}, w(\cdot)\right]>0
$$

for all $t \in[0, T]$ and all $x_{0} \neq 0$.

The optimization problem (6.8) subject to the constraint defined by (2.1) is a linear quadratic optimal control problem. Using a result from linear quadratic optimal control theory (see [20]), we conclude that (6.8) implies that the Riccati differential equation (5.2) with terminal condition $Y(T)=0$ has a defined solution over $[0, T]$ such that $Y(0)>0$. This completes the proof of the first part of the theorem.

(ii) $\Rightarrow$ (i) We have shown that $x_{0} \in X_{0}\left[\left.u_{0}(\cdot)\right|_{0} ^{T},\left.y_{0}(\cdot)\right|_{0} ^{T}, d\right]$ if and only if there exists an input $w(\cdot) \in \mathcal{L}_{2}[0, T]$ such that $J \leq d$, with $J$ defined by (6.7). Now consider the following minimization problem:

$$
\min _{w(\cdot) \in \mathcal{L}_{2}[0, T]} J\left[x_{0}, w(\cdot)\right]
$$

where the minimum is taken over all $x(\cdot)$ and $w(\cdot)$ connected by (2.1) with the condition $x(0)=x_{0}$. This problem is a linear quadratic optimal tracking problem in forward time. To convert this tracking problem into a standard tracking problem, we decompose the state vector $x(t)$ into $x(t)=\tilde{x}(t)+x_{1}(t)$ where $x_{1}(t)$ is the solution to the state equation (3.12), and hence, $\widetilde{x}(t)$ satisfies the state equation (3.13). Therefore, the cost function (6.7) can be written as

$$
\begin{aligned}
J\left[x_{0}, w(\cdot)\right]= & \tilde{J}\left[\widetilde{x}_{0}, w(\cdot)\right]=\int_{0}^{T}\left\{w(t)^{\prime} Q(t) w(t)\right. \\
& -\left\|K(t)\left[\widetilde{x}(t)+x_{1}(t)\right]+G(t) u_{0}(t)\right\|^{2} \\
& +\left(y_{0}(t)-C(t)\left[\tilde{x}(t)+x_{1}(t)\right]\right)^{\prime} R(t)\left(y_{0}(t)\right. \\
& \left.\left.-C(t)\left[\tilde{x}(t)+x_{1}(t)\right]\right)\right\} d t
\end{aligned}
$$

It can be observed that (3.13) and (6.10) define a standard linear quadratic tracking problem with $y_{0}(\cdot), u_{0}(\cdot)$ and $x_{1}(\cdot)$ all treated as reference inputs. Indeed, the only difference between the standard linear quadratic optimal tracking problem considered in [21, Section 3.6] and the one considered here is that in our case the cost function (6.10) is sign indefinite. However, if the matrix $Y(\cdot)$ is the solution of the Riccati equation (6.2), then the infimum in (6.9) will be achieved for any $\tilde{x}_{0}, u_{0}(\cdot)$ and $y_{0}(\cdot)$. Indeed, as in the proof of Theorem 5.1 , we can write

$$
\min _{w(\cdot) \in \mathcal{L}_{2}[0, T]} J\left[\widetilde{x}_{0}, w(\cdot)\right]=\tilde{x}_{0}^{\prime} Y(0) \tilde{x}_{0}-2 \tilde{x}_{0}^{\prime} \nu(0)+m(0)
$$


where $\nu(\cdot)$ and $m(\cdot)$ are solutions of the differential equations

$$
\begin{aligned}
-\dot{\nu}(t)= & {\left[A(t)-B_{1}(t) Q(t)^{-1} B_{1}(t)^{\prime} Y(t)\right]^{\prime} \nu(t) } \\
& +C(t)^{\prime} R(t) y_{0}(t)-\left[C(t)^{\prime} R(t) C(t)\right. \\
& \left.-K(t)^{\prime} K(t)\right] x_{1}(t)+K(t)^{\prime} G(t) u_{0}(t) ; \quad \nu(T)=0
\end{aligned}
$$

$$
\begin{aligned}
-\dot{m}(t)= & {\left[y_{0}(t)-C(t) x_{1}(t)\right]^{\prime} R(t)\left[y_{0}(t)-C(t) x_{1}(t)\right] } \\
& -\left\|G(t) u_{0}(t)+K(t) x_{1}(t)\right\|^{2} \\
& -\nu(t)^{\prime} B_{1}(t) Q(t)^{-1} B_{1}(t)^{\prime} \nu(t) ; \quad m(T)=0
\end{aligned}
$$

for $t \in[0, T]$. Now, let us define

$$
\phi(t)=\nu(t)+Y(t) x_{1}(t)
$$

and

$$
s(t)=m(t)+2 x_{1}(t)^{\prime} \phi(t)-x_{1}(t)^{\prime} Y(t) x_{1}(t) .
$$

It is straightforward, but tedious to show that $\phi(\cdot)$ satisfies (6.3) and $s(\cdot)$ satisfies

$$
\begin{aligned}
\dot{s}(t)= & -y_{0}(t) R(t) y_{0}(t)+u_{0}(t)^{\prime} G(t)^{\prime} G(t) u_{0}(t) \\
& +\phi(t)^{\prime} B_{1}(t) Q(t)^{-1} B_{1}(t)^{\prime} \phi(t)+2 u_{0}(t) B_{2}(t) \phi(t)
\end{aligned}
$$

for $t \in[0, T]$ and with $s(T)=0$. Moreover, it can be verified that

$$
\min _{w(\cdot) \in \mathcal{L}_{2}[0, T]} J\left[x_{0}, w(\cdot)\right]=x_{0}^{\prime} Y(0) x_{0}-2 x_{0}^{\prime} \phi(0)+s_{0} .
$$

Hence, we can conclude that the set

$$
\begin{aligned}
X_{0} & {\left[\left.u_{0}(\cdot)\right|_{0} ^{T},\left.y_{0}(\cdot)\right|_{0} ^{T}, d\right] } \\
& =\left\{x_{0} \in \mathbf{R}^{n}: \min _{w(\cdot) \in \mathcal{L}_{2}[0, T]} J\left[x_{0}, w(\cdot)\right] \leq d\right\}
\end{aligned}
$$

is as given in (6.4). This completes the proof of the theorem.

\section{ILLUSTRATIVE EXAMPLE}

In this section, we present an example to illustrate our main results. The example consists of an oscillator with uncertainty in the frequency of oscillation and uncertainty in the measurement equation. The uncertain system is described as follows:

$$
\begin{aligned}
& \dot{x}(t)=\left[\begin{array}{rr}
0 & 1 \\
-2 & 0
\end{array}\right] x(t)+\left[\begin{array}{l}
0 \\
1
\end{array}\right] w(t) \\
& z(t)=\left[\begin{array}{cc}
1 & 0 \\
0.1 & 0.1
\end{array}\right] x(t) \\
& y(t)=\left[\begin{array}{ll}
1 & -1
\end{array}\right] x(t)+v(t) .
\end{aligned}
$$

The uncertainty in this system is assumed to satisfy the integral quadratic constraint (2.4) with $R=1, Q=1, d=1, x_{0}=0$, and

$$
X_{0}=\left[\begin{array}{cc}
10 & 0 \\
0 & 0.04
\end{array}\right] .
$$

In the first part of the example we consider a robust prediction problem. We also assume that the integral quadratic constraint holds for $T=7.5$. To illustrate the performance of our predictor applied to this uncertain system, we consider the uncertainties to be such that $w(t)=\left[\begin{array}{ll}1 & 0\end{array}\right] x(t)$, and $v(t)=$ $-\left[\begin{array}{ll}0.1 & 0.1\end{array}\right] x(t)$ and $x(0)=\left[\begin{array}{ll}0 & 1\end{array}\right]^{\prime}$. It is straightforward to verify that this uncertainty satisfies the integral quadratic

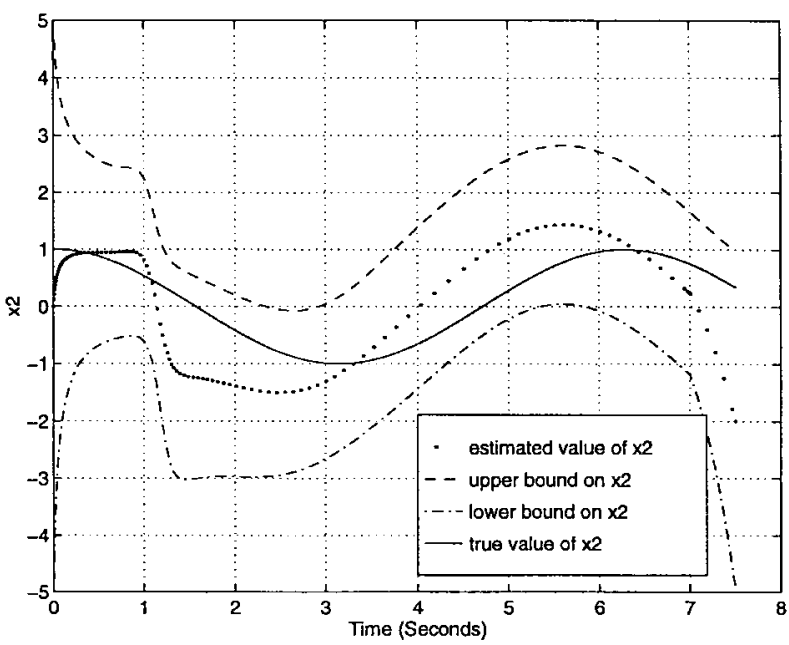

Fig. 6. Estimated value of $x_{2}$ as a function of time.

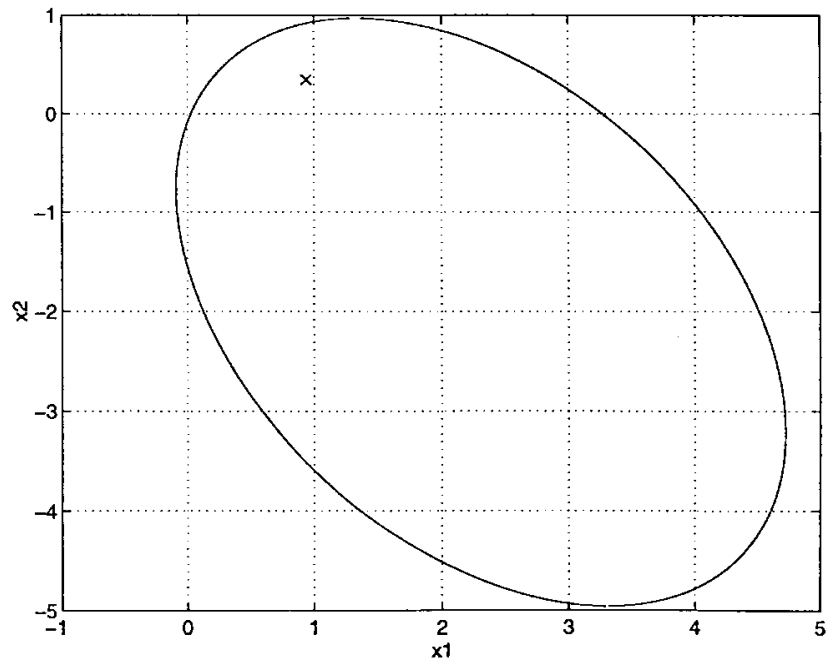

Fig. 7. Ellipsoidal bound for $x(7.5)$.

constraint. Corresponding to this uncertainty realization is the linear system

$$
\begin{aligned}
& \dot{x}(t)=\left[\begin{array}{rr}
0 & 1 \\
-1 & 0
\end{array}\right] x(t) ; \\
& y(t)=\left[\begin{array}{ll}
0.9 & -1.1
\end{array}\right] x(t) .
\end{aligned}
$$

Note that system (7.1) is of the form (2.1) with $u(\cdot) \equiv 0$.

Now, we assume that $T=7.5$ and $q=0.5 \mathrm{~s}$. Therefore, the measurements up to $T-q=7 \mathrm{~s}$ are available. Fig. 6 shows the resulting estimate of the state variable $x_{2}$, upper and lower bounds on the value of $x_{2}$, and the true value of $x_{2}$ as a function of time over the interval $[0,7.5]$. Also, in Fig. 7, we show the ellipsoidal bounding set of the form (4.4) bounding the true value of the system at time $t=7.5$.

The second part of the example is concerned with robust observability of the uncertain system (7.1). We assume that the integral quadratic constraint (6.1) is satisfied with the above values of $Q, R$, and $d$ and with $T=10$. Hence, the system (7.2) can be used to generate the measure output $\left.y_{0}(\cdot)\right|_{0} ^{10}$. The objective is to determine the set of possible initial conditions $X_{0}\left[\left.y_{0}(\cdot)\right|_{0} ^{10}, 1\right]$ for the above uncertain system based on the 


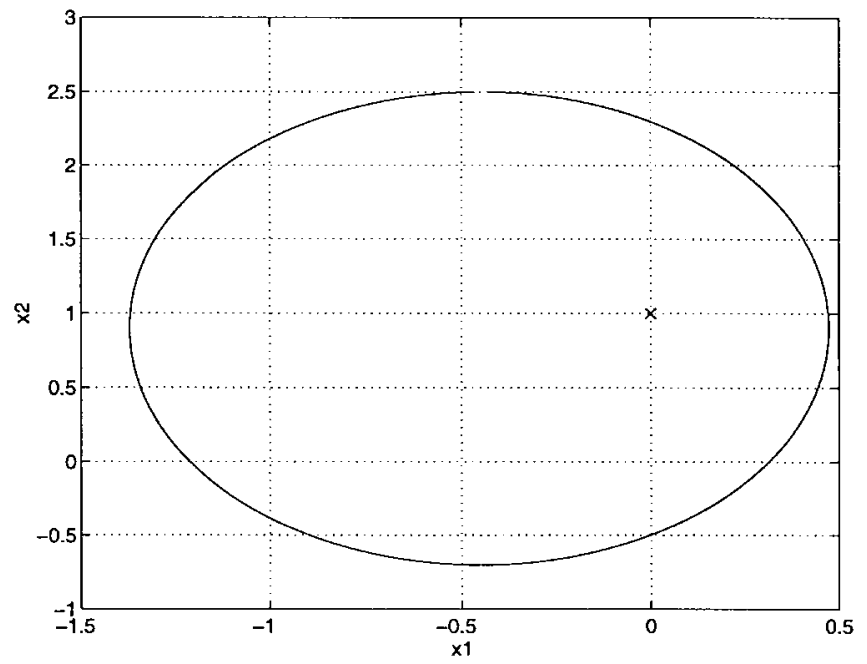

Fig. 8. Ellipsoidal bound for $x(0)$.

output measurements $\left.y_{0}(\cdot)\right|_{0} ^{10}$. This set can be calucated using the result of Theorem 6.1. Indeed, in Fig. 8, we show the ellipsoidal bounding set of the form (6.4) bounding the true value of the initial condition $x(0)$.

\section{REFERENCES}

[1] R. G. Brown and P. Y. C. Hwang, Introduction to Random Signals and Kalman Filtering. New York: Wiley, 1992, vol. 2.

[2] Y. Theodor, U. Shaked, and C. E. de Souza, "A game theory approach to robust discrete-time $H_{\infty}$ estimation, IEEE Trans. Signal Processing, pp. 1486-1495, 1994.

[3] B. N. Jain, "Guaranteed error estimation in uncertain systems," IEEE Trans. Automat. Contr., vol. AC-20, pp. 230-232, 1975.

[4] C. E. de Souza, M. Fu, and L. Xie, " $H^{\infty}$ estimation for discrete-time linear uncertain systems," Int. J. Robust and Nonlinear Contr., vol. 1, no. 2, pp. 111-123, 1991.

[5] L. Xie, C. E. de Souza, and M. D. Fragoso, " $H^{\infty}$ filtering for linear periodic systems with parameter uncertainty," Syst. Contr. Lett., vol. 17, pp. 343-350, 1991.

[6] I. R. Petersen and D. C. McFarlane, "Optimal guaranteed cost control and filtering for uncertain linear systems," IEEE Trans. Automat. Contr., vol. 39, pp 1971-1977, 1994.

[7] C. E. de Souza, U. Shaked, and M. Fu, "Robust $H_{\infty}$ filtering for continuous time varying uncertain systems with deterministic input signals," IEEE Trans. Signal Processing, vol. 43, pp. 709-719, 1995.

[8] M. Fu, C. E. de Souza, and L. Xie, " $H^{\infty}$ estimation for uncertain systems," Int. J. Robust and Nonlinear Contr., vol. 2, pp. 87-105, 1992.

[9] A. V. Savkin and I. R. Petersen, "Recursive state estimation for uncertain systems with an integral quadratic constraint," IEEE Trans. Automat. Contr., vol. 40, pp. 1080-1083, 1995.

[10] K. M. Nagpal and P. P. Khargonekar, "Filtering and smoothing in an $H_{\infty}$ setting," IEEE Trans. Automat. Contr., vol. AC-36, pp. 152-166, 1991.

[11] Y. Theodor, "Robust $H_{\infty}$ and $H_{2}$ estimation and control," Ph.D. dissertation, Tel-Aviv Univ., 1994.

[12] T. Basar and P. Bernhard, " $H_{\infty}$-Optimal Control and Related Minimax Design Problems: A Dynamic Game Approach. Boston, MA: Birkhäuser, 1991.

[13] K. Zhou, J. C. Doyle, and K. Glover, Robust and Optimal Control. Englewood Cliffs, NJ: Prentice-Hall, 1996.

[14] I. R. Petersen and D. C. McFarlane, "Robust state estimation for uncertain systems," in Proc. 30th Conf. Decision and Control, Brighton, U.K., 1991.

[15] _ _ "Optimal guaranteed cost filtering for uncertain discrete time systems," Int. J. Robust and Nonlinear Contr., vol. 6, no. 4, pp. 267-280, 1996.

[16] D. P. Bertsakas and I. B. Rhodes, "Recursive state estimation for a setmembership description of uncertainty," IEEE Trans. Automat. Contr., vol. AC-16, pp. 117-128, 1971.

[17] A. V. Savkin and I. R. Petersen, "A new approach to model validation and fault diagnosis," in Proc. 3rd Eur. Control Conf., Rome, Italy, 1995, pp. $1418-1422$.
[18] I. R. Petersen and D. W. J. Pulle, "Robust Kalman filtering in direct torque control," in 7th Eur. Conf. Power Electronics and Applications, 1997.

[19] A. V. Savkin and I. R. Petersen, "Model validation for robust control of uncertain systems with an integral quadratic constraint," Automatica, vol. 32, no. 4, pp. 603-606, 1996.

[20] D. J. Clements and B. D. O. Anderson, Singular Optimal Control: The Linear Quadratic Problem. Berlin, Germany: Springer-Verlag, 1978.

[21] F. L. Lewis, Optimal Control. New York: Wiley, 1986.

[22] M. Green and D. J. N. Limebeer, Linear Robust Control. Englewood Cliffs, NJ: Prentice-Hall, 1994.

[23] F. L. Lewis, Optimal Estimation. New York: Wiley, 1986.

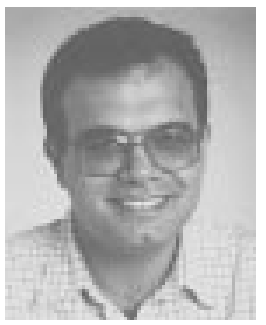

S. O. Reza Moheimani (M'98) was born in Shiraz, Iran, in 1967. He received the B.Sc. degree from Shiraz University in 1990 and the M.Eng.Sc. and $\mathrm{Ph} . \mathrm{D}$. degrees from The University of New South Wales in 1993 and 1996, all in electrical and electronic engineering.

Following his graduation, he was employed by the school of Electrical and Electronic Engineering, Australian Defence Force Academy, Campbell, as a Senior Research Associate. In August 1997, he joined the Department of Electrical and Computer Engineering, University of Newcastle, NSW, Australia, as a Research Academic. He is interested in robust filtering and control of uncertain systems, industrial applications of robust control, applications of Linear Matrix Inequalities in robust control theory, active control of noise and vibrations, smart structures, and signal processing.

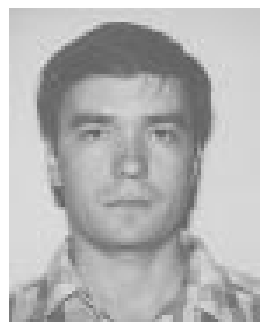

Andrey V. Savkin was born in Norilsk, U.S.S.R, in 1965. He received the M.S. degree in mathematics and the $\mathrm{Ph} . \mathrm{D}$. degree in applied mathematics from the Leningrad State University, U.S.S.R, in 1987 and 1991, respectively.

From 1987 to 1992, he worked in the All-Union Television Research Institute, Leningrad, U.S.S.R From 1992 to 1994, he held a post-doctoral position in the Department of Electrical Engineering, Australian Defence Force Academy, Canberra. From 1994 to 1996, he was a Research Fellow with the Department of Electrical and Electronic Engineering and the Cooperative Research Center for Sensor Signal and Information Processing at the University of Melbourne, Australia. Since 1996, he has been a Senior Lecturer with the Department of Electrical and Electronic Engineering at the University of Western Australia, Nedlands. His current research interests include robust control and filtering, $\mathrm{H}$-infinity control, nonlinear systems, model validation, hybrid dynamical systems, image processing, and financial mathematics. $\mathrm{He}$ is an Associate Editor for Stability and Control: Theory and Applications.

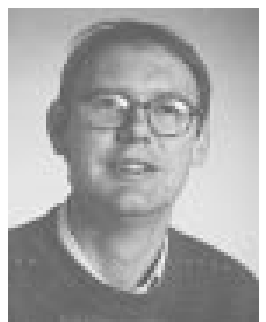

Ian R. Petersen (S'80-M'83-SM'96) was born in Victoria, Australia, in 1956. He received the Bachelor of Engineering (Electrical) degree from the University of Melbourne in 1979 and the M.S and Ph.D. degrees from the University of Rochester Rochester, NY.

From 1983 to 1985, he was a Post-Doctoral Fellow in the Department of Systems Engineering, Australian National University. In 1985, he was appointed as a Lecturer in the Department of Electrical Engineering, Australian Defence Force Academy, and he is currently an Associate Professor in this department. In 1989, he was a visitor in the Department of Engineering, Cambridge University. He served as an Associated Editor for the IEEE TRANSACTIONS ON AutOMATIC ConTroL and Systems and Control Letters. Currently he is an Associate Editor for Automatica. His main research interests are in robust control theory, $H^{\infty}$ control, robust filtering, and optimal control theory. 